\title{
Neutrino properties deduced from the double beta decay study
}

\author{
Sabin Stoica*t \\ International Center for Advanced Training and Research in Physics, P.O. Box MG12, 077125 \\ Bucharest-Magurele, Romania \\ E-mail: sabin.stoicadcifra.infim.ro \\ Ovidiu Nitescu \\ International Center for Advanced Training and Research in Physics, P.O. Box MG12, 077125 \\ Bucharest-Magurele, Romania \\ E-mail: nitescu.ovidiuvasiledgmail.com
}

Double beta decay (DBD) is a rare nuclear process of great interest due to its potential to provide information about physics beyond the Standard Model (BSM). For example, the discovery of the neutrinoless double-beta $(0 v \beta \beta)$ decay mode could give answers to fundamental issues about lepton number conservation, $\mathrm{CP}$ and Lorentz symmetry violation in the weak sector, and about still unknown neutrino properties such as: are neutrinos Dirac or Majorana particles?; neutrino absolute masses; what is the correct hierarchy of the neutrino masses?; are there sterile neutrinos?, etc. Theoretically, the first important stage in the DBD study consists in the derivation of half-lives formulas and precisely computation of the nuclear matrix elements (NME) and phase space factors (PSF) entering these formulas, for different decay modes and transitions to ground or excited states of the daughter nuclei. Reliable computations of these quantities result in reliable predictions of DBD half-lives and constrains of the BSM parameters related to the possible mechanisms that may contribute to the $0 v \beta \beta$ decay. In this paper we give first a short review of the theoretical challenges in the computation of the NME and PSF for double-beta decay. Then we present a new, more consistent, approach to calculate these quantities, namely to calculate directly their product.

The 39th International Conference on High Energy Physics (ICHEP2018)

4-11 July, 2018

Seoul, Korea

\footnotetext{
* Speaker.

${ }^{\dagger}$ This work is supported by the Romanian Ministry of Research and Innovation through the Program 5N/2018, project PN18-110201
} 


\section{Introduction}

Double-beta decay (DBD) is the rarest spontaneous nuclear decay measured until now which attracts much interest due to its potential to provide information about BSM physics [四]-[B]]. It may occurs through several decay modes classified in two categories i) decays in which two antineutrinos/neutrinos are emitted in the final states besides the two electrons/positrons $(2 v \beta \beta)$, which conserve the lepton number and ii) decays in which no neutrinos/anti-neutrinos are emitted $0 v \beta \beta$, which occurs with lepton number violation (LNV) and are allowed only in theories more general than the SM. Until now there are experimental measurements for fourteen double-beta decays, for $0 v \beta \beta$ decay, until now, there is no convinced experimental evidence. If any $0 v \beta \beta$ decay mode will be discovered, this would provide us important information related to, at least the following fundamental issues: a) lepton number conservation; b) $\mathrm{CP}$ and Lorentz symmetry violation in the weak sector c) neutrino character: Dirac or Majorana?; d) hints on absolute neutrino mass and mass hierarchy; e) possible existence of right-handed currents in the weak interaction; f) hints on possible mechanisms that can contribute to the occurrence of $0 \mathrm{v} \beta \beta$ decay; g) possible existence of heavy (sterile) neutrinos, etc.

\section{Theoretical challenges in the DBD study}

In a good approximation the half-life formulas can be expressed as product of phase space factors (PSF) and nuclear matrix elements (NME) [䧃]-[ []]

$$
\left(T_{1 / 2}^{2 v}\right)^{-1}=G^{2 v}\left(E_{0}, Z\right) g_{A}^{4}\left|m_{e} c^{2} M^{2 v}\right|^{2} ;\left(T_{1 / 2}^{0 v}\right)^{-1}=G^{0 v}\left(E_{0}, Z\right) g_{A}^{4}\left|M_{l}^{0 v}\right|^{2}\left(\left\langle\eta_{l}\right\rangle\right)^{2}
$$

where $G^{2 v}, G^{0 v}$ are the PSF, $M^{2 v}$ and $M^{0 v}$ are the NME, for the $2 v, 0 v$ decay modes. In the case of $0 v \beta \beta$ an additional factor $\left\langle\eta_{l}\right\rangle$ appears, which is related to the specific mechanism 1 that can contribute to this decay mode. We note that the above formulas are written such that the product of the nuclear (NME) and atomic part (PSF) is expressed in $\left[y r^{-1}\right]$, and the axial-vector constant $\left(g_{A}\right)$ to the forth power is separated from the other components. Such forms of the DBD half-lives allow an easier using of the theoretical results for interpreting the DBD data and the possibility to investigate uncertainties on $g_{A}$ by exploiting data from different DBD experiments and decay modes. As seen, for estimating/predicting DBD lifetimes and deriving beyond SM parameters, a precise, reliable computation of both the PSF and NME is mandatory. The largest uncertainties in the DBD calculations come from the NME. They are computed with different nuclear methods,

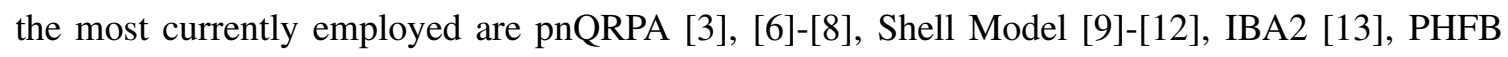
[14]], generator coordinate method [ㄷ]. These methods differ each other mainly by the choice of the model spaces and type of correlations taken into account in calculation. The errors in the NME computation associated with each method have been extensively debated in the literature over

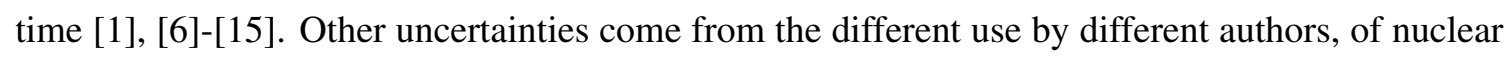
approximations (inclusion of short-range correlations, finite nuclear size, higher order terms in nucleon currents, etc.) and different values of some input parameters like nuclear radius, form factors and axial-vector constant, involved in calculation. Particularly important is the $g_{A}$ value (which can be 1.0 = quark value; 1.273 = free nucleon value; or other quenched value $\tilde{\text { (0.4-0.9) }}$ 
because there is a strong dependence of the half-lives on this constant. The errors coming from the different choice of values of these parameters can increase significantly the uncertainty in the half-lives computation, hence appropriate attention should also be paid to this source or errors. An analysis of the size of errors associated with these uncertainties can be found in [ए6]. Because there are still large differences between the NME values reported in literature by different groups, their computation still remains a challenge in the study of the DBD process.

The other important quantities in the calculation of the DBD half-lives are the PSF. Until the recent past they were considered to be computed with enough accuracy [B] , [20]. However, newer calculations [䧃]-[鴫], [प्प] performed with more rigorous methods, i.e. by using exact electron Dirac wave functions (w.f.), improving the way of taking into account the finite nuclear size (FSN) and electron screening effects, and the use of more realistic form of the Coulomb potential, revealed notable differences of the PSF values as compared with older results, especially for heavier nuclei, for positron emitting and $E C$ decay modes and for transitions to excited states. The errors in the PSF computation can come either from i) the method of calculation of the electron w.f., namelynon-relativistic approach [177]-[ए8]]; -relativistic approach with approximate electron w.f. [B]]; relativistic approach with exact electron w.f. [䧃]-[可], [ए耳]; or ii) the numerical accuracy both in resolution of the Dirac equations for getting the electron radial functions and the integration of the PSF expressions. We also re-computed PSFs and reported the results in [[5],[ए耳], with a new code that improves the numerical accuracy both in solving the Dirac equation for obtaining the electron distorted wave functions and in the integration of the PSF expressions. Moreover, we used a Coulomb-type potential built up from a realistic proton density distribution in the daughter nucleus, by solving the Schrödinger equation for a Woods-Saxon (WS) potential.

\section{Calculation of products $N M E \times P S F$ for double-beta decay}

A more consistent way to calculate the NME and PSF quantities was proposed recently in [2]], namely to calculate directly their product. The products for the $2 v$ and $0 v$ decay modes are defined as follows:

$$
P^{2 v}=G^{2 v} \times\left|m_{e} c^{2} M^{2 v}\right|^{2} ; P^{0 v}=G^{0 v} \times\left|M_{l}^{0 v}\right|^{2}
$$

The detailed expressions of these products can be found in [2]

For computing the products $P^{2 v}$ and $P^{0 v}$ we build up numerical codes taking advantage of our

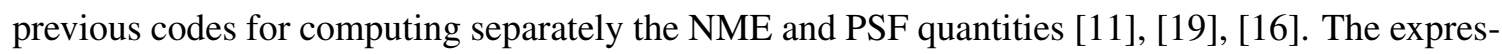
sions of the products $P^{(2,0) v}$ contain factors outside the integrals stemming from the multiplication and simplification of factors that multiply separately the nuclear and kinetic parts. Also, their kinetic part (phase space factors) and the nuclear part (NME) have common input parameters as $R_{A}$, $\left\langle E_{N}\right\rangle$ and $g_{A}$. In Table 1 we calculated the $P^{(2,0) v}$ for five experimentally most interesting nuclei. With these values the calculation of half-lives is directly and without any confusion regarding the units.

\section{Conclusions}

We presented some challenges encountered in the calculation of nuclear matrix elements and phase space factors, two key quantities entering the DBD half-lives. We described the possible 
Table 1: Results for $2 v \beta \beta$ decay mode

\begin{tabular}{lcc} 
Nucleus & $P^{2 v}\left[y r^{-1}\right]$ & $P^{0 v}\left[y r^{-1}\right]$ \\
\hline${ }^{48} \mathrm{Ca}$ & $123.81 \times 10^{-21}$ & $7.30 \times 10^{-15}$ \\
${ }^{76} \mathrm{Ge}$ & $5.16 \times 10^{-21}$ & $9.95 \times 10^{-15}$ \\
${ }^{82} \mathrm{Se}$ & $186.62 \times 10^{-21}$ & $34.45 \times 10^{-15}$ \\
${ }^{130} \mathrm{Te}$ & $25.26 \times 10^{-21}$ & $71.45 \times 10^{-15}$ \\
${ }^{136} \mathrm{Xe}$ & $20.30 \times 10^{-21}$ & $71.01 \times 10^{-15}$ \\
\hline
\end{tabular}

source of errors that can bring uncertainty in their values and suggested another way of calculating them, namely by computing directly their product in a single formula. Such an approach has advantages in terms of the consistency of using the same approximations and values for the input parameters that enter into the separate formulas of the two quantities.

Acknowledgments. This work was support by the Romanian Ministry of Research and Innovation through Program Nucleus 5N/2018, project PN18-110201.

\section{References}

[1] J. Vergados, H. Ejiri and F. Simkovic, Rep. Prog. Phys. 75, 106301 (2012).

[2] F. T. Avignon, S.R. Elliott and J. Engel, Rev. Mod. Phys. 80, 481 (2008).

[3] J. Suhonen and O. Civitarese, Phys. Rep. 300, 123 (1998).

[4] J. Kotila and F. Iachello, Phys. Rev. C 85, 034316 (2012).

[5] S. Stoica and M. Mirea, Phys. Rev. C 88, 037303 (2013).

[6] M. Kortelainen and J. Suhonen, Phys. Rev. C 75, 051303 (2007); Phys. Rev. C 76, 024315 (2007).

[7] F. Simkovic, A. Faessler, H. Muther, V. Rodin, and M. Stauf, Phys. Rev. C 79, 055501 (2009).

[8] S. Stoica and H.V. Klapdor-Kleingrothaus, Nucl. Phys. A 694, 269 (2001).

[9] E. Caurier, J. Menendez, F. Nowacki, and A. Poves, Phys. Rev. Lett. 100, 052503 (2008).

[10] J. Menendez, A. Poves, E. Caurier, F. Nowacki, and A. Poves, Nuclear Physics A 818, 139 (2009).

[11] M. Horoi, S. Stoica and B.A. Brown, Phys. Rev. C (2007).

[12] M. Horoi and S. Stoica, Phys. Rev. 81, 024321-024328 (2010).

[13] J. Kotila and F. Iachello, Phys. Rev. C 87, 024313 (2013).

[14] P.K. Rath, R. Chandra, K. Chaturvedi, P.K. Raina, J.G. Hirsch, Phys. Rev. C 82, 064310 (2010).

[15] T.R. Rodriguez and G. Martinez-Pinedo, Phys. Rev. Lett 105, 252503 (2010).

[16] A. Neacsu and S. Stoica, J.Phys. G 41, 015201 (2014).

[17] H. Primakov and S.P. Rosen, Rep. Prog. Phys. 22, 121 (1959).

[18] W.C. Haxton and G.J. Stephenson Jr., Prog. Theor. Part. Nucl. Phys. 12, 409 (1984).

[19] M. Mirea, T.E. Pahomi, S. Stoica, Rom. Rep. Phys. 67, 872 (2015).

[20] T. Tomoda, Rep. Prog. Phys. 54, 53 (1991).

[21] S. Stoica, Chinese Phys. C (accepted). 